\title{
Tumour-derived adhesion factor in colorectal cancer
}

\author{
ANDERS MÄLARSTIG ${ }^{1}$, DICK WÅGSÄTER ${ }^{1}$, STURE LÖFGREN ${ }^{2}$, \\ ANDERS HUGANDER ${ }^{3}$, NIKLAS ZAR ${ }^{3}$ and JAN DIMBERG ${ }^{4}$ \\ ${ }^{1}$ Atherosclerosis Research Unit, Department of Medicine, Karolinska Institute, SE-171 76 Stockholm; \\ Departments of ${ }^{2}$ Clinical Microbiology, and ${ }^{3}$ Surgery, Ryhov County Hospital; ${ }^{4}$ Department of Natural Science \\ and Biomedicine, University College of Health Sciences, SE-551 11 Jönköping, Sweden
}

Received July 1 2009; Accepted August 21, 2009

DOI: $10.3892 / \mathrm{mmr} 000000200$

\begin{abstract}
Tumour-derived adhesion factor (TAF) has been shown to be associated with breast, prostate and colorectal cancer (CRC), acting as tumour suppressor or tumour promoter by mechanisms not as yet understood. Here, we comparatively analyzed the expression profile of TAF in plasma, tumour and paired normal tissue from patients with CRC. In addition, we investigated the relationship between TAF and systemic inflammation, mirrored by the elevation of interleukin-6 (IL-6) and TAF levels in plasma. Levels of TAF and IL- 6 were determined by ELISA. Immunohistochemistry was performed to investigate the site of TAF expression. We also used a TaqMan system to investigate a TAF single nucleotide polymorphism (rs2041437) with a potential effect on CRC. TAF protein levels were significantly $(\mathrm{P}<0.001)$ higher in colorectal tumours than in normal tissue, and were increased in patients with Dukes' stages B and $\mathrm{C}$ compared to A. Immunohistochemistry revealed heterogeneous TAF expression mainly in the epithelial cells of the cancer and normal tissue. The plasma TAF level was reduced in CRC patients compared with the controls $(\mathrm{P}=0.002)$, independent of the inflammatory marker IL-6. Regarding genotype and allelic distributions, significant differences between CRC patients and control subjects or associations between clinical characteristics and TAF levels in tissue and plasma were not observed. In conclusion, altered TAF protein expression in cancer tissue may be a potential biomarker in colorectal carcinogenesis. Further research exploring the regulation of TAF is required to evaluate whether TAF is linked to clinical outcome.
\end{abstract}

\section{Introduction}

Insulin-like growth factors (IGFs) including IGF-1 and IGF-2 have been reported to play a key role in cell proliferation,

Correspondence to: Dr Jan Dimberg, Department of Natural Science and Biomedicine, University College of Health Sciences, Box 1026, SE-551 11 Jönköping, Sweden

E-mail: jan.dimberg@hhj.hj.se

Key words: tumour-derived adhesion factor, protein expression, plasma, biomarker anti-apoptosis and mitogenesis (1). These factors have been implicated in the development of colorectal cancer (CRC), and their effects are regulated in part by insulin-like growth factor binding proteins (IGFBPs), which have both low and high affinity for IGFs (1). Studies have suggested that circulating levels of IGFs may be related to an increased risk of prostate (2), lung (3) and colorectal $(4,5)$ cancer. Overall, the bioavailability and thereby the bioactivity of IGFs in circulation may be due to the plasma concentration of IGFBPs. Additionally, an increase in serum IGF-1 and decrease in serum IGFBP-3 may be associated with the development of CRC (6). However, case control studies linking the relationship between IGF-1, IGFBP-3 and various types of cancer showed that higher concentrations of circulating IGF-1 were associated with CRC, while high circulating levels of IGFBP-3 did not protect from CRC (7).

Tumour-derived adhesion factor (TAF), also known as IGFBP-related protein 1 (IGFBP-rP1), is widely expressed in various tissues, including lung, brain, prostate and gastrointestinal tract tissues (8). Epithelial cells in the duodenum, ileum and colon show weak to moderate immunoreactivity, and there are indications that pathological conditions leading to inflammatory response may increase TAF expression $(9,10)$.

In certain human cancer cell lines, such as breast and prostate cancer cell lines, the expression of TAF is decreased $(11,12)$. On the other hand, immunohistochemistry has revealed higher expression of TAF in CRC compared to paired noncancerous tissue $(13,14)$. Patients with positive staining for TAF have been shown to have a favorable prognosis compared to patients with negative staining for TAF (13). This, however, contradicts the results of Adachi et al (15).

To date, no studies have focused on gene variants of TAF in relation to CRC. In an ongoing study, we investigated a TAF single nucleotide polymorphism (SNP) of rs2041437 (C $\rightarrow$ T) located upstream of the promoter in inflammatory disease of the large vessels. This polymorphism has exhibited associations with inflammatory markers (unpublished data).

The aim of the current study was to examine plasma concentrations and the protein expression of TAF in CRC patients, and to investigate the relationship between TAF and systemic inflammation, as mirrored by the elevation of interleukin- 6 (IL-6) levels. We also aimed to investigate the potential effect of the TAF gene polymorphism rs2041437 on CRC. 


\section{Materials and methods}

Study population. The study included blood samples from 274 consecutive patients from southeastern Sweden, collected during surgical resection for primary colorectal adenocarcinomas at the Department of Surgery, Ryhov County Hospital, Jönköping, Sweden. The clinicopathological characteristics of the patients were determined from surgical and pathological records. The patient group included 144 males and 130 females with a mean age of 70 years (range 29-93 years). The tumours were localized in the colon $(n=140)$ and rectum $(n=134)$, and were classified according to the Dukes' classification system: stage A $(n=50)$, stage B $(n=114)$, stage $C(n=92)$ and stage $D(n=18)$. Blood donors $(n=278)$ with no known CRC history from the same geographical region as the CRC patients were selected as controls. The control group consisted of 146 males and 132 females with a mean age of 68 years (range 50-83 years). The blood samples were centrifuged to separate plasma and blood cells, and then stored at $-70^{\circ} \mathrm{C}$. The study was approved by the local ethics committee, and informed consent was obtained from each subject.

DNA extraction and genotype determination. DNA was isolated from blood using the QiaAmp DNA Blood Kit (Qiagen, CA, USA). The DNA samples were genotyped using the 5'-exonuclease allelic discrimination assay (Applied Biosystems, CA, USA). The Taqman SNP Genotyping assay was used for analysis of the rs2041437 genotype (Applied Biosystems). DNA (10 ng) was amplified in a total volume of $12 \mu \mathrm{l}$ containing 1X TaqMan Universal PCR Master Mix (Applied Biosystems) included in the SNP Genotyping assay. Amplification was performed with an initial cycle at $50^{\circ} \mathrm{C}$ for 2 min followed by 1 cycle at $95^{\circ} \mathrm{C}$ for $10 \mathrm{~min}$ and finally 40 cycles at $95^{\circ} \mathrm{C}$ for $15 \mathrm{sec}$, then $60^{\circ} \mathrm{C}$ for $1 \mathrm{~min}$. A post-PCR endpoint reading was performed on each plate using the 7500 Fast RealTime PCR System (Applied Biosystems). The manual calling option in the allelic discrimination application ABI PRISM 7500 SDS (version 1.3.1) was used to assign the genotypes.

Tissue samples and lysates. Tissue samples from 93 CRC patients were used in the study. The tumours and matched normal mucosa were from 53 males and 40 females with a mean age of 67 years (range 29-89 years). The tumours were localized in the colon $(n=52)$ and rectum $(n=41)$, and were classified according to the Dukes' classification system: stage A $(n=17)$, stage B $(n=34)$, stage $C(n=29)$ and stage $D(n=13)$. Tumour tissue and adjacent normal mucosa $(\sim 5 \mathrm{~cm}$ from the tumour) from each patient were excised and immediately frozen at $-70^{\circ} \mathrm{C}$ until analysis.

Frozen tumour tissue and normal mucosa were thawed, homogenized in ice cold lysis buffer containing PBS (9.1 mM dibasic sodium phosphate, $1.7 \mathrm{mM}$ monobasic sodium phosphate, $150 \mathrm{mM} \mathrm{NaCl}, \mathrm{pH}$ 7.4) and $1 \%$ Nonidet P-40, $0.5 \%$ sodium deoxycholate, $0.1 \%$ sodium dodecyl sulphate (SDS), $100 \mu \mathrm{g} / \mathrm{ml}$ phenylmethylsulphonyl flouride (PMSF), $2 \mu \mathrm{g} / \mathrm{ml}$ aprotinin, $1 \mathrm{mM}$ sodium orthovanadate and $1 \mu \mathrm{g} / \mathrm{ml}$ leupeptin. The lysate was placed on ice for $30 \mathrm{~min}$ and then centrifuged at $13,000 \mathrm{x}$ g for $10 \mathrm{~min}$. The protein content of the supernatant fluid was determined for each sample using the Bradford protein assay (Bio-Rad Laboratories, UK).
Plasma samples. One hundred and three CRC patients and 108 blood-control donors were available for plasma collection. Patient blood was collected before surgery and all blood, including that of the controls, was separated by centrifugation within $1 \mathrm{~h}$. Plasma was removed and stored at $-70^{\circ} \mathrm{C}$. The CRC patient group comprised 60 males and 43 females with a mean age of 68 years (range 29-82 years). The tumours were categorized according to Dukes' classification: stage A ( $n=22)$, stage $B(n=41)$, stage $C(n=35)$ and stage $D(n=5)$. Forty-seven tumours were located in the rectum and 56 in the colon. Controls consisted of plasma from 58 males and 50 females with a mean age of 62 years (range 55-68 years).

ELISA for TAF and IL-6. For the measurement of TAF in tissue and plasma, an enzyme-linked immunosorbent (ELISA) assay was developed using goat TAF capture antibody (R\&D Systems Europe, Abingdon, UK) for coating of the ELISA plate (Costar, Corning Inc., Corning, NY, USA) at $4^{\circ} \mathrm{C}$ overnight. After blocking the plates in carbonate buffer (50 mM, pH 9.5) with $1 \% \mathrm{BSA}$ for $1 \mathrm{~h}$ at room temperature, the samples were added. Recombinant human TAF (R\&D Systems) was used to obtain a standard curve. After washing the plate in PBS with $0.1 \%$ BSA and $0.1 \%$ Tween, biotinylated TAF detection antibody (R\&D Systems) was added. Streptavidin-HRP (R\&D Systems) reagent was added, followed by the addition of 3,3',5,5'tetramethylbenzidine (TMB) substrate (Serva Electrophoresis, GmbH, Heidelberg, Germany). IL-6 in plasma was measured using a commercially available ELISA kit (R\&D Systems) following the manufacturer's instructions. The tissue levels of TAF in the tumour and paired normal tissue were expressed as nanograms per milligram (ng/mg) of protein, and the plasma TAF and IL- 6 concentrations from CRC patients and control subjects were expressed as nanograms per millilitre (ng/ml) and picograms per millilitre $(\mathrm{pg} / \mathrm{ml})$, respectively.

Immunohistochemistry. Immunohistochemical staining was used to study the cell type origin of the TAF expression. Staining was performed using a standard protocol on $4-\mu \mathrm{m}$ sections from formalin-fixed paraffin-embedded tissue blocks. Antigen retrieval was performed by microwave treatment in citrate buffer ( $\mathrm{pH}$ 6.0). Endogenous peroxidase activity was quenched by treatment with $3 \%$ hydrogen peroxide for $5 \mathrm{~min}$. Sections were subsequently incubated with a primary goat anti-TAF antibody (R\&D Systems) overnight at $4^{\circ} \mathrm{C}$, and then with biotinylated horse anti-goat secondary antibody (ImmunKemi, Järfälla, Sweden). Avidin-biotin peroxidase complexes (ImmunKemi) were added, followed by visualization with 3,3-diaminobenzidine tetrahydrochloride (ImmunKemi). Sections were counterstained with Mayer's haematoxylin (Histolab Products, Göteborg, Sweden). As a negative control, the primary antibody was replaced by a goat isotype control IgG (Santa Cruz Biotechnology Inc., Santa Cruz, CA, USA).

Statistical analysis. Data were expressed as the median and interquartile range. A Q-Q plot of the expected normal distribution against the observed values indicated that the distribution of plasma concentrations of TAF and IL-6 were skewed. Therefore, these variables were log-transformed prior to statistical analysis. For the comparison of soluble TAF or 
IL-6 concentrations between controls and cases or between the different Dukes' stages, analyses of variance (ANOVA) were used. Differences in TAF protein concentration between tumour and normal paired tissues was tested using the paired t-test. Correlations were analyzed using the Pearson correlation test on log-transformed variables.

Differences in the frequencies of the TAF gene polymorphism between CRC patients and the control group and between clinical characteristics within the CRC subgroup were analyzed using the $\chi^{2}$ test, and the Hardy-Weinberg equilibrium was tested for the genotypes. Statistical analysis was performed using the SPSS for Windows computer package (Rel. 15.0, 2006; SPSS Inc., Chicago, IL, USA). Results were considered significant at $\mathrm{P}<0.05$.

\section{Results}

Protein levels of TAF in tissue. ELISA was used to evaluate TAF protein expression in CRC tissue and in paired normal tissue collected $5 \mathrm{~cm}$ from the tumour. Protein lysates from 93 CRC patients were used.

A significant up-regulation $(\mathrm{P}<0.001)$ of TAF was found in CRC tissue specimens [3.90 (2.80-5.25) $\mathrm{ng} / \mathrm{mg}$ protein] in comparison with paired normal tissue [2.72 (2.17-3.42) ng/mg] (Fig. 1). An assessment of the relative expression (tumour vs. normal tissue) showed that $78.5 \%$ (73/93) of the cases were up-regulated. To assess the results according to disease severity, the CRC patients were further divided into subgroups according to Dukes' stage. The results showed that lysates from the tumour site of patients with Dukes' stages B, $\mathrm{C}$ and $\mathrm{D}$ had higher TAF levels compared to the paired normal tissue. Additionally, lysates from the tumour site of patients with Dukes' stages B and C had higher TAF expression than those from Dukes' stage A patients (Fig. 1). No association was found with other clinical characteristics, such as tumour location, age and gender (data not shown).

Plasma concentrations of TAF and IL-6. To evaluate the TAF and IL- 6 concentrations in plasma, ELISA was used. Plasma from 103 CRC patients and 108 controls was used. As IL-6 is considered to reflect inflammatory response, the plasma concentration of IL- 6 was also determined by ELISA. A significantly $(\mathrm{P}=0.002)$ lower concentration of TAF was observed in plasma from patients $[25.6(21.1-56.2) \mathrm{ng} / \mathrm{ml}]$ in comparison with the plasma from controls [37.5 (21-161) ng/ml] (Fig. 2). However, the TAF plasma concentration of CRC patients did not reflect the tissue concentration, and was not related to Dukes' stage, tumour location, age or gender (data not shown).

In agreement with other studies, a higher IL-6 plasma concentration was observed in CRC patients [10 (4-22) pg/ml] compared to the controls [1.1 (1.1-2) $\mathrm{pg} / \mathrm{ml}]$, with a statistical difference between these groups $(\mathrm{P}<0.001)$. However, IL-6 was not significantly correlated with plasma TAF in CRC patients or in controls (data not shown).

Immunohistochemical findings. Immunohistochemistry was performed in order to detect the site of TAF expression. Immunostaining of TAF was mainly evident in the epithelial cells of the tumour and in epithelial cells at the resection border reflecting normal tissues. Staining was heterogenous, with an

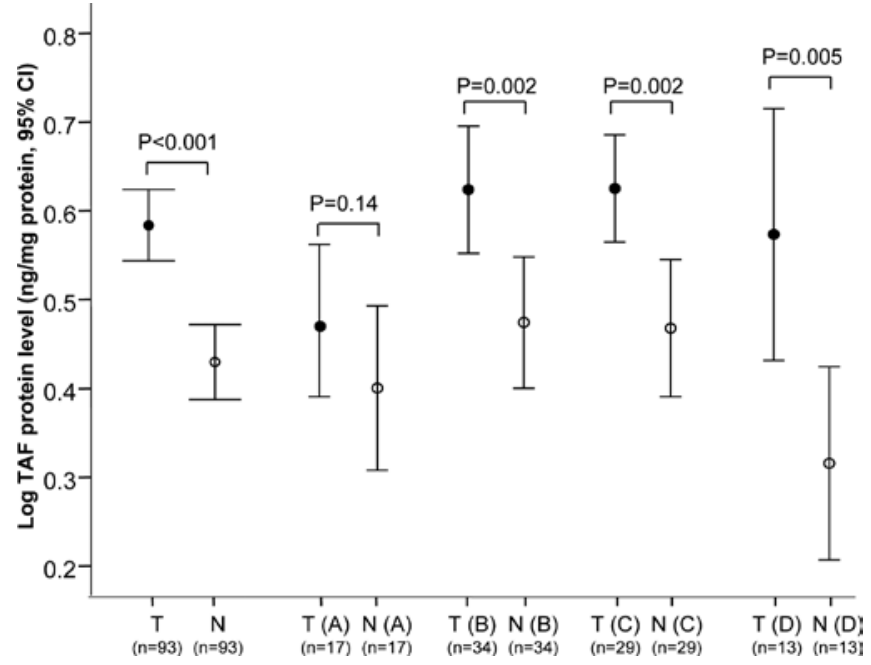

Figure 1. Log TAF protein level expressed as $\mathrm{ng} / \mathrm{mg}$ protein $(95 \% \mathrm{CI})$ in colorectal cancer tissue $(\mathrm{T})$ and paired normal tissue $(\mathrm{N})$, divided according to Dukes' stage (A-D).

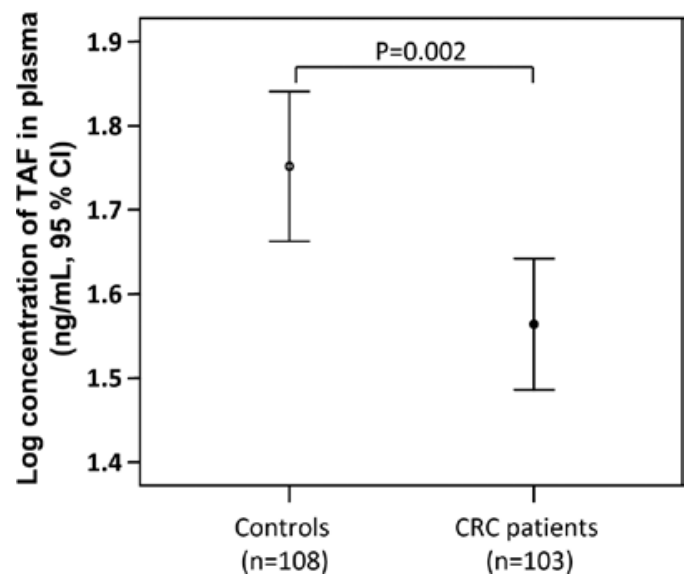

Figure 2. Log TAF concentration in plasma from CRC patients and controls expressed as $\mathrm{ng} / \mathrm{ml}(95 \% \mathrm{CI})$.

intensity varying from none to strong (Fig. 3). No staining was observed in an isotypic IgG antibody used as a negative control (data not shown).

TAF gene polymorphism. To investigate the influence of the gene variant TAF rs2041437 $(\mathrm{C} \rightarrow \mathrm{T})$ on colorectal carcinogenesis as well as its correlation with TAF expression, the SNP was examined in $276 \mathrm{CRC}$ patients and 278 controls. There were no significant differences in genotype distribution or allelic frequency between the CRC patients and controls (Table I). Significant differences also failed to be observed upon subdividision of the patients into groups of colonic and rectal cancer or localized Dukes' A + B and disseminated Dukes' $\mathrm{C}+\mathrm{D}$ disease (Table II). Genotype and allelic distributions in $\mathrm{CRC}$ patients and the control group were not associated with other clinical characteristics, such as age and gender or TAF levels in tissue or plasma (data not shown). Neither the patient nor the control group showed significant deviation from HardyWeinberg equilibrium. 

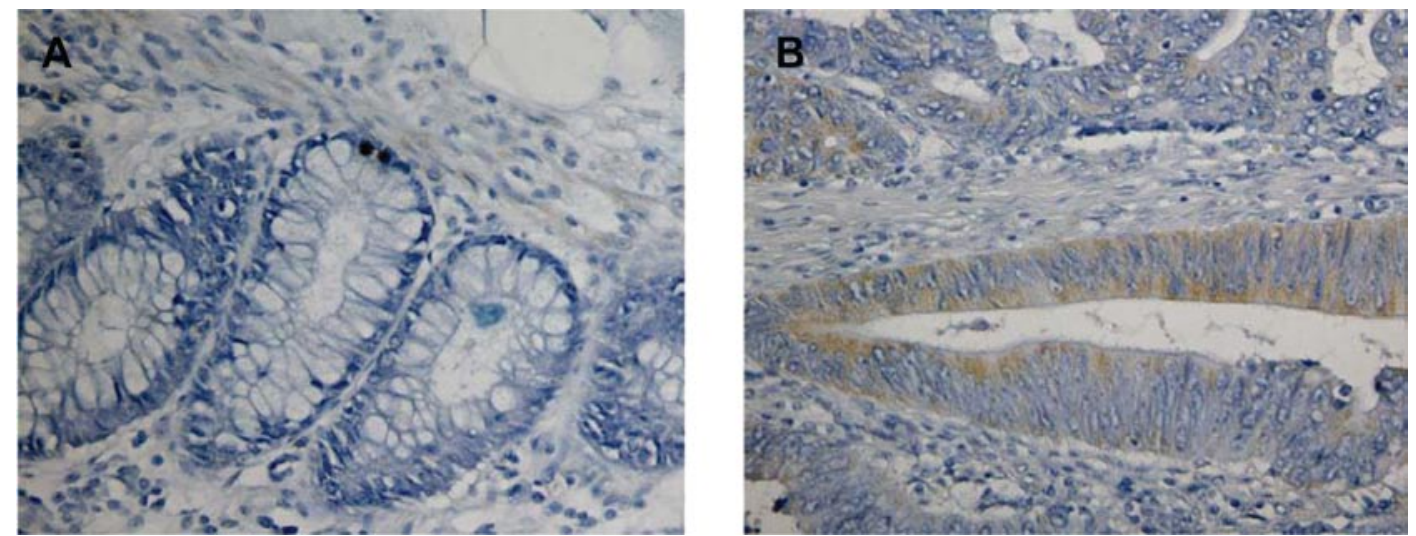

Figure 3. Immunohistochemical detection and localization of TAF in colorectal tissue from patients with colorectal cancer. Detection of TAF in (A) normal and (B) cancerous tissue. Magnification x200.

Table I. Genotypic and allelic distributions in the \% (n) CRC patients and controls with the TAF gene polymorphism rs 2041437.

\begin{tabular}{lccccc}
\hline Genotype & CRC $(\mathrm{n}=274)$ & Controls $(\mathrm{n}=278)$ & Allele & CRC $(\mathrm{n}=548$ alleles $)$ & Controls $(\mathrm{n}=556$ alleles $)$ \\
\hline $\mathrm{C} \rightarrow \mathrm{T}$ & $38.7(106)$ & $41.4(115)$ & $\mathrm{C}$ & $63.3(347)$ & $62.6(348)$ \\
$\mathrm{C} / \mathrm{C}$ & $49.3(135)$ & $42.4(118)$ & $\mathrm{T}$ & $36.7(201)$ & $37.4(208)$ \\
$\mathrm{C} / \mathrm{T}$ & $12.0(33)$ & $16.2(45)$ & & &
\end{tabular}

CRC patients vs. controls, not statistically significant.

Table II. Genotype and allele numbers of the TAF gene polymorphism rs2041437 and the clinicopathological characteristics of the CRC patients.

\begin{tabular}{|c|c|c|c|c|c|}
\hline & \multicolumn{3}{|c|}{ Genotype } & \multicolumn{2}{|c|}{ Allele } \\
\hline & $\mathrm{C} / \mathrm{C}$ & $\mathrm{C} / \mathrm{T}$ & $\mathrm{T} / \mathrm{T}$ & $\mathrm{C}$ & $\mathrm{T}$ \\
\hline \multicolumn{6}{|l|}{ Tumour site } \\
\hline Rectum $(n=134)$ & 53 & 63 & 18 & 169 & 99 \\
\hline Colon $(n=140)$ & 53 & 72 & 15 & 178 & 102 \\
\hline \multicolumn{6}{|l|}{ Dukes' stage } \\
\hline$A+B(n=164)$ & 67 & 72 & 25 & 206 & 122 \\
\hline$C+D(n=110)$ & 39 & 63 & 8 & 141 & 79 \\
\hline
\end{tabular}

Colon vs. rectum and Dukes' A + B vs. Dukes' C + D, not statistically significant.

\section{Discussion}

IGF-1 and IGF-2 have been implicated in the development of colorectal cancer (CRC). Their effects are regulated in part by insulin-like growth factor binding proteins (IGFBPs), which have both low and high affinity for IGFs (1). Overall, the bioavailability and thereby the bioactivity of IGFs may be due to the level of IGFBPs. Studies suggest that an increase in serum IGF-1 and a decrease in serum IGFBP-3 may be associated with the development of CRC (6). TAF or
IGFBP-related protein 1 is widely expressed in various organs including the lungs, brain, prostate and gastrointestinal tract (8). In certain human cancer cell lines, such as breast and prostate cancer cell lines, the expression of TAF is decreased $(11,12)$. However, immunohistochemistry studies have revealed increased expression of TAF in CRC compared to paired non-cancerous tissue, and it has been suggested that TAF plays a tumour suppressor role in colorectal carcinogenesis $(13,14)$ and indicates a favorable prognosis in comparison with patients with negative staining for TAF (13). However, these 
findings contradict the results of Adachi et al, who found TAF expression to be correlated with a poor prognosis (15).

In this study, Swedish CRC patients assessed by ELISA were demonstrated to have a significantly higher level of TAF protein in cancer tissues in comparison with paired normal tissues. This is consistent with data from studies using an immunohistochemistry approach $(13,14)$.

To the best of our knowledge, this study is the first to analyse considerable quantities of protein lysates prepared from CRC and paired normal tissue. We found a significant difference in terms of disease stage and TAF expression. Thus, the expression of TAF may reflect disease progression. To further characterize TAF in CRC, immunohistochemistry was used to evaluate the site of TAF expression. Immunostaining of TAF was heterogenous and mostly seen in the epithelial cells of the cancer and normal tissue.

Various lines of evidence indicate that TAF has both stimulatory and inhibitory effects on CRC progression $(13,14)$. The molecular association of TAF with colorectal tumour progression is as yet unknown. However, it has been suggested that TAF may play an important role in vascular biology, and appears to be involved in angiogenesis occurring in tumour tissues (16). In addition, it is possible that TAF modifies the bioavailability of IGFs. This has been reported to play an important role in cell proliferation, anti-apoptosis and mitogenesis (1).

We also investigated circulating levels of TAF in patients with CRC. In one analysis, the concentration of TAF did not differ significantly between CRC patients $(n=40)$ and control subjects $(n=62)(9)$. In our larger sample collection of patients $(n=103)$ and controls $(n=108)$, significantly lower plasma TAF concentrations were observed in CRC patients compared to control subjects, and circulating levels of TAF in the patients were not associated with tumour TAF concentration and clinical characteristics such as Dukes' stage, tumour localization and gender. Our findings indicate that plasma TAF levels do not reflect the local level of TAF in cancerous tissue. This discrepancy may in part be explained by the fact that several tissues and organs (8) can potentially express and release TAF, thereby disguising local changes in TAF expression. Moreover, the potential sources may alter the expression of TAF under pathological conditions. Based on indications that inflammatory conditions may increase TAF $(9,10)$, we also investigated circulating levels of the pro-inflammatory cytokine IL-6, which is elevated in CRC (17). In the present study, we confirmed that plasma IL-6 levels were significantly higher in the CRC patients than in the controls, but were not correlated with plasma TAF. The TAF concentration in plasma does not appear to be correlated with systemic inflammatory response reflected by the inflammatory marker IL-6. Further research to clarify the relationship between IL-6 and TAF is required.

To date, no studies have focused on gene variants of TAF in relation to CRC. In an ongoing study of the TAF gene polymorphism $\mathrm{rs} 2041437(\mathrm{C} \rightarrow \mathrm{T})$ in relation to vascular inflammation, the SNP shows an association with inflammatory markers. Since CRC is associated with an increase in inflammatory markers such as IL-6, we investigated whether this SNP could be a potential candidate affecting colorectal carcinogenesis. Despite the fact that this SNP showed no association with TAF expression or CRC outcome, there are several other potential candidate SNPs in the TAF gene.

In conclusion, TAF may have potential as a biomarker in colorectal carcinogenesis, as TAF expression was found to be increased in CRC tissue. However, the mechanisms behind this have yet to be understood. Further research exploring whether TAF acts as a tumour suppressor or promoter is needed. It is possible to relate our existing data on the TAF expression profile of CRC patients to the influence of TAF on a 3- or 5-year survival rate. Our data thereby add to a growing list of investigations on the influence of TAF in various types of cancer.

\section{Acknowledgements}

Grants were received from Futurum - The Academy of Healthcare, County Council of Jönköping, Sweden, from the University College of Health Sciences, Jönköping, Sweden, and from the Nanna Svartz Foundation. The authors thank Karin Ström (Ryhov County Hospital, Jönköping) for excellent technical support.

\section{References}

1. Durai R, Yang W, Gupta S, Seifalian AM and Winslet MC: The role of the insulin-like growth factor system in colorectal cancer: review of current knowledge. Int J Colorectal Dis 20: 203-220, 2005.

2. Shi R, Berkel $\mathrm{HJ}$ and $\mathrm{Yu} \mathrm{H}$ : Insulin-like growth factor-1 and prostate cancer: a meta-analysis. Br J Cancer 85: 991-996, 2001.

3. Yu H, Spitz MR, Mistry J, Gu J, Hong WK and Wu X: Plasma levels of insulin-like growth factor-I and lung cancer risk: a casecontrol analysis. J Natl Cancer Inst 91: 151-156, 1999.

4. Probst-Hensch NM, Yuan JM, Stanczyk FZ, Ross RK and Yu MC: IGF-1, IGF-2 and IGFBP-3 in prediagnostic serum: association with colorectal cancer in a cohort of Chinese men in Shanghai. Br J Cancer 85: 1695-1699, 2001.

5. Kaaks R, Toniolo P, Akhmedkhanov A, Lukanova A, Biessy C, Dechaud H, Rinaldi S, Zeleniueh-Jacquotte A, Shore RE and Riboli E: C-Peptide, insulin-like growth factor (IGF)-I, IGFbinding proteins and colorectal cancer risk in women. J Natl Cancer Inst 92: 1592-1600, 2000.

6. Nomura AM, Stemmermann GN, Lee J and Pollak MN: Serum insulin-like growth factor I and subsequent risk of colorectal cancer among Japanese-American men. Am J Epidemiol 158: 424-431, 2003.

7. Renehan AG, Zwahlen M, Minder C, O'Dwyer ST, Shalet SM and Egger M: Insulin-like growth factor (IGF)-I, IGF binding protein-3 and cancer risk: systemic review and metaregression analysis. Lancet 363: 1346-1353, 2004.

8. Degeorges A, Wang F, Frierson HF, Seth A and Sikes RA: Distribution of IGFBP-rP1 in normal human tissues. J Histochem Cytochem 48: 747-754, 2000

9. Kutsukake $\mathbf{M}$, Ishihara $\mathrm{R}$, Momose $\mathrm{K}$, Isaka $\mathrm{K}$, Itokazu $\mathrm{O}$, Higuma C, Matsutani T, Matsuda A, Sasajima K, Hara T and Tamura K: Circulating IGF-binding protein 7 (IGFBP7) levels are elevated in patients with endometriosis or undergoing diabetic hemodialysis. Reprod Biol Endocrinol 6: 54, 2008.

10. Lopez-Bermejo A, Khosravi J, Fernandez-Real JM, Hwa V, Pratt KL, Casamitjana R, Garcia-Gil MM, Rosenfeld RG and Ricart W: Insulin resistance is associated with increased serum concentration of IGF-binding protein-related protein 1 (IGFBP-rP1/mac25). Diabetes 55: 2333-2339, 2006.

11. Burger AM, Zhang X, Li H, Ostrowski JL, Beatty B, Venanzoni M, Papas T and Seth A: Down-regulation of T1A12/mac25 a novel insulin-like growth factor binding protein related gene is associated with disease progression in breast carcinomas. Oncogene 16: 2459-2467, 1998.

12. Hwa V, Tomasini-Sprenger C, Bermejo AL, Rosenfeld RG and Plymate SR: Characterization of insulin-like growth factor-binding protein-related protein 1 in prostate cells. J Clin Endocrinol Metab 83: 4355-4362, 1998. 
13. Ruan W, Xu E, Xu F, Ma Y, Deng H, Huang Q, Lv B, Hu H, Lin J, Cui J, Di M, Dong J and Lai M: IGFBP7 plays a potential tumor suppressor role in colorectal carcinogenesis. Cancer Biol Ther 6: 354-359, 2007.

14. Shao L, Huang Q, Luo M and Lai M: Detection of the differentially expressed gene IGF-binding protein-related protein- 1 and analysis of its relationship to fasting glucose in Chinese colorectal cancer patients. Endocr Relat Cancer 11: 141-148, 2004.

15. Adachi Y, Itoh F, Yamamoto H, Arimura Y, Kikkawa-Okabe Y, Miyazaki K, Carbone DP and Imai K: Expression of angiomodulin (tumor-derived adhesion factor/mac25) in invading tumor cells correlates with poor prognosis in human colorectal cancer. Int J Cancer 95: 216-222, 2001.
16. Lopez-Bermejo A, Khosravi J, Corless CL, Krishna RG, Diamandi A, Bodani U, Kofoed EM, Graham DL, Hwa V and Rosenfeld RG: Generation of anti-insulin-like growth factor-binding protein-related protein 1 (IGFBP-rP1/MAC25) monoclonal antibodies and immunoassay: quantification of IGFBP-rP1 in human serum and distribution in human fluids and tissues. J Clin Endocrinol Metab 88: 3401-3408, 2003.

17. Chung YC and Chang YF: Serum interleukin-6 levels reflect the disease status of colorectal cancer. J Surg Oncol 83: 222-226, 2003. 